\title{
Linear Gas Sensor for Methane based on a Selectively Permeable Membrane
}

\author{
M. Bartholmai ${ }^{1}$, S. Ebert ${ }^{1}$, P.P. Neumann ${ }^{1}$, R. Noske ${ }^{1}$, W. Rehak ${ }^{2}$, D. Lazik ${ }^{3}$ \\ BAM Federal Institute for Materials Research and Testing, Berlin, Germany \\ matthias.bartholmai@bam.de \\ ${ }^{2}$ Optotransmitter-Umweltschutz-Technologie e.V. (OUT e.V.), Berlin, Germany \\ ${ }^{3}$ Helmholtz Centre for Environmental Research - UFZ, Halle, Germany
}

\begin{abstract}
:
In preliminary work, gas sensors in linear form based on the measuring principle of gas selective permeability through a membrane were developed and introduced for the detection and quantification of gas concentrations. In this study, first experimental results are presented for adapting the technology for measuring methane $\left(\mathrm{CH}_{4}\right)$. A material with suitable selective permeability was identified and utilized in a sensor setup containing the gas selective membrane and a reference membrane, both integrated in a measuring cell, to which a gas stream with defined $\mathrm{CH}_{4}$ concentrations was applied. The results prove the sensor's capability for measuring methane and indicate further application potential of the method, e.g., as a robust field monitoring technology, since $\mathrm{CH}_{4}$ is the major component of natural gas, town gas, and fracking gas.
\end{abstract}

Key words: selective permeability, quantification of gas concentrations, spatially distributed monitoring, gas storage sites, methane measuring

\section{Introduction}

Numerous applications require gas monitoring with spatial coverage in linear or plane format. Typical examples are gas storage sites, pipelines, and disposals.

Conventional gas sensors measure punctual and are sensitive only for the target gas in their immediate vicinity. Linear gas sensors are implemented in form of flexible tubular membranes and measure relevant gas concentrations along their total length [1]. A current field study is carried out to validate such sensors for a spatially distributed monitoring of subsurface $\mathrm{CO}_{2}$ gas storage sites under application conditions using a test field with dimension of $400 \mathrm{~m}^{2}$ surface area [2], [3] [4].

\section{Measuring Principle}

The measuring principle of linear membranebased gas sensors is based on the selective permeation of gases through a polymer membrane. The measuring method [5] combines the gas specific diffusion rates through such a membrane with Dalton's law of partial pressures. It enables the calculation of gas concentrations based on the ideal gas law by using measurements of pressure, time, and temperature. The measurement signal of this approach is the time derivation of the pressure $a_{1}$ [mbar/s] near the dynamic equilibrium through the membrane, which allows for a much faster gas detection compared to a detection using a membrane based phase separating module, where partition equilibrium has to be achieved through the membrane.

\section{Sensor Implementation}

The sensitive component of such linear gas sensors is the membrane material, which has to be selectively permeable for the target gas. A material with suitable selectivity for measuring $\mathrm{CH}_{4}$ is polydimethylsiloxane (PDMS). It has to be taken into account that PDMS is also selectively permeable for $\mathrm{CO}_{2}$, i.e., resulting in a cross-sensitivity. But in this study, the primary focus was on demonstrating the general capability to measure $\mathrm{CH}_{4}$. Nevertheless, many applications address gas mixtures mainly constituted by $\mathrm{CO}_{2}$ and $\mathrm{CH}_{4}$ (natural gas, town gas, and fracking gas) and offer practical interest in a single line sensor for leakage detection with sensitivity to both components.

In cooperation between BAM and UFZ, a test setup was developed featuring a linear sensor consisting of two PDMS tubes and two impermeable tubes as reference membrane (Fig. 1). All tubular membranes were integrated in a measuring cell, to which a gas flow with defined $\mathrm{CH}_{4}$ concentrations was applied. 


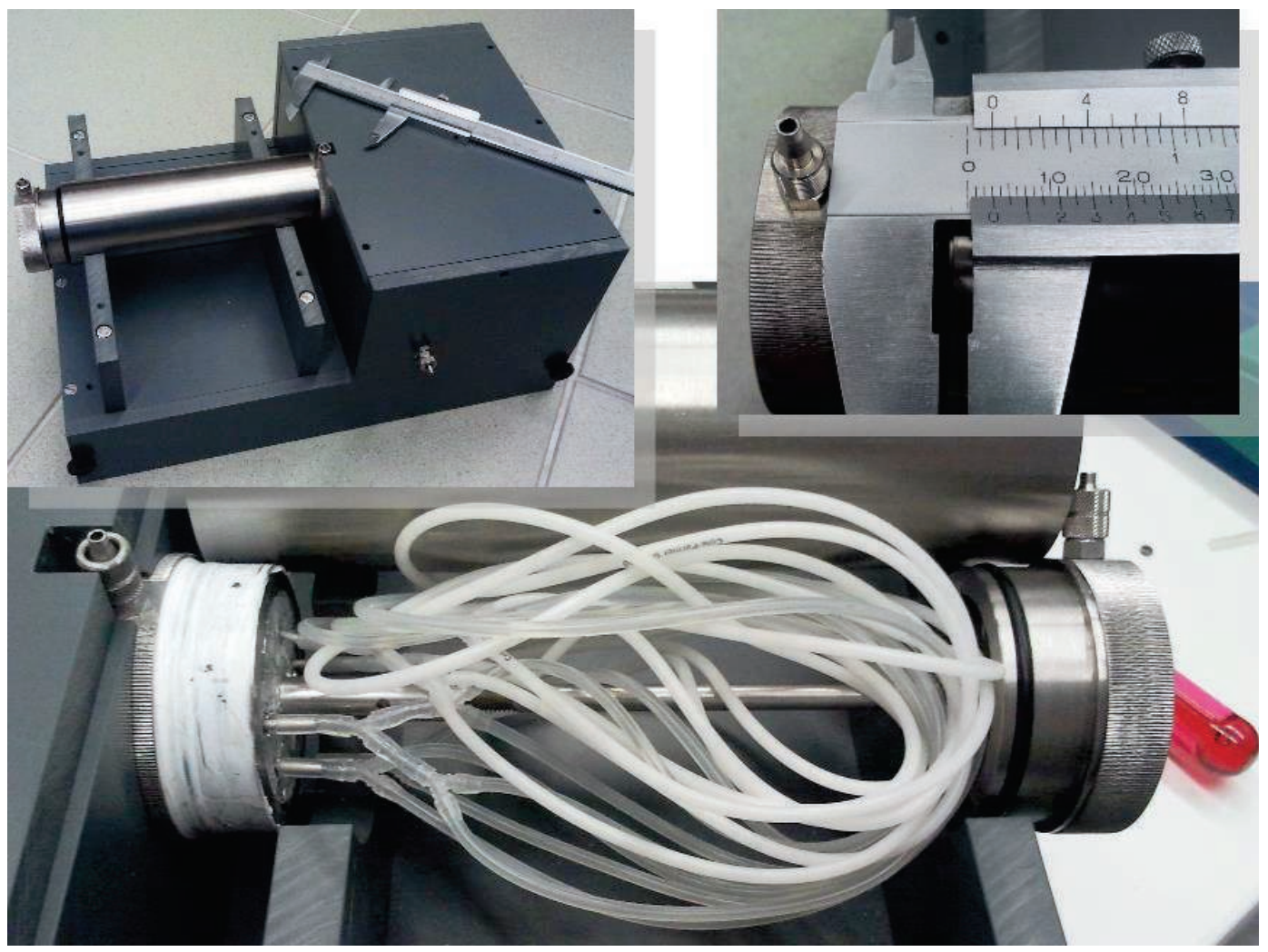

Fig. 1: Sensor setup containing the gas selective PDMS membrane and a reference membrane, integrated in the measuring cell, to which the gas stream was applied.
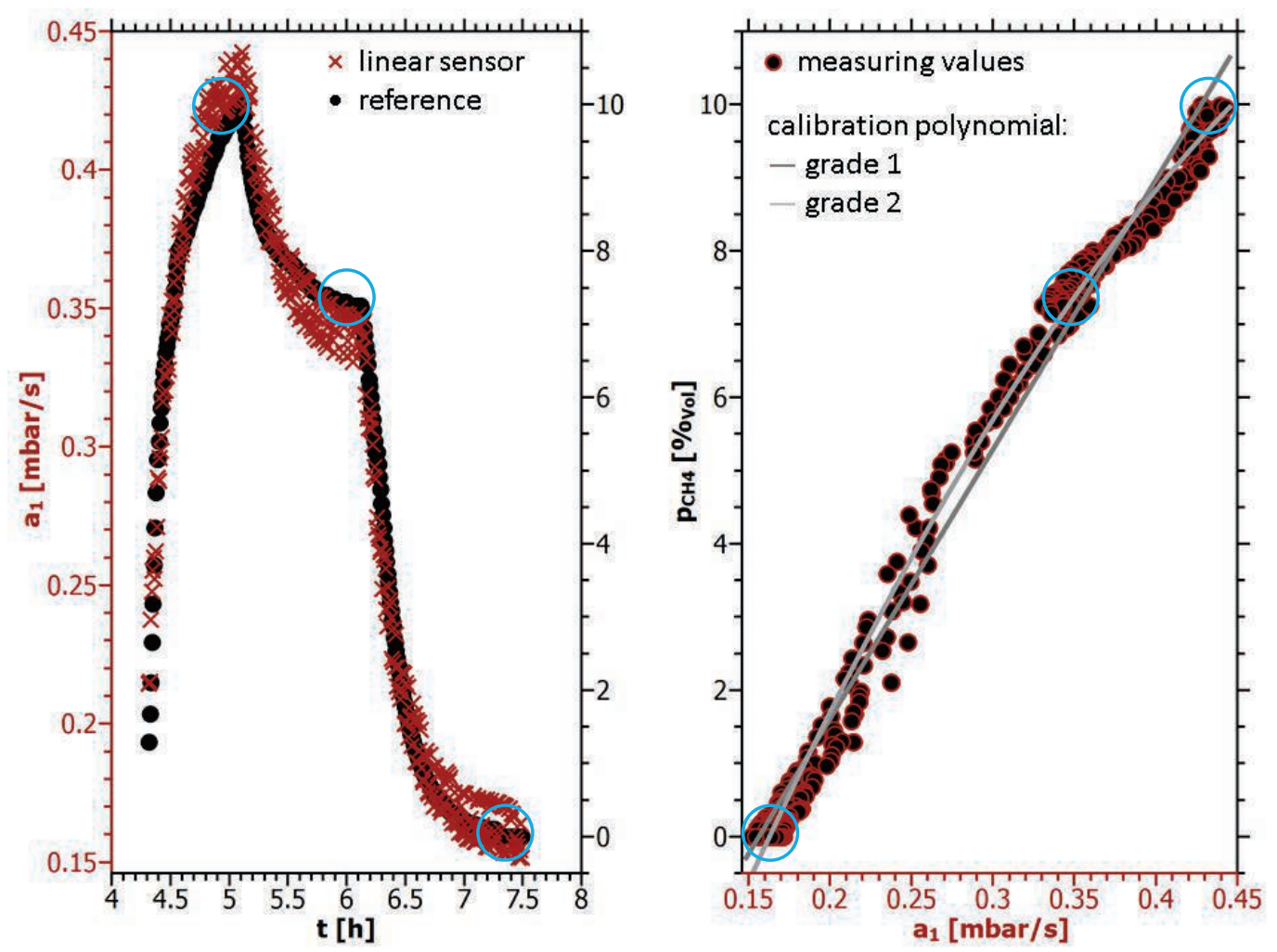

Fig. 2: Measured methane signal - linear sensor (red) with results in mbar/s and reference sensor (black) with results in \%ol. Both results were used to calculate a calibration function for the linear sensor, which is shown right. 


\section{Results und Outlook}

Fig. 2 displays the comparison of measuring results between the linear sensor and a reference sensor (commercially available catalytic sensor). Both signals show comparable progression and build the basis for calculation of a calibration function. The significantly higher noise level of the linear sensor can be attributed to several effects:

- a time delay between sensor and reference response due to the spatial separation of sensor and reference,

- fluctuations of the initial conditions for measurement of $a_{1}$,

- use of primary non-stacked and nonfiltered measurement values of $a_{1}$ during processing,

- dynamic changes of temperature and humidity in the gas stream, which were not considered or compensated in the simple experimental setup.

The calibration functions which are shown in Fig. 2, right, were approximated by a linear function or quadratic polynomial using eq. (1).

$$
p_{C H 4}=\sum_{i=0}^{n} k_{i} \cdot a_{1}^{i}
$$

Fig. 2, left, shows a relative overshooting of the membrane based gas sensor, which can be attributed to different response characteristics of sensor and reference. Taking into account only measurements with low dynamics (slow change of concentration) improves the accuracy of the calculated calibration functions. This is demonstrated for corresponding measurements (blue circles in Fig. 2, right and left).

Tab. 1: Calibration parameters and deviation

\begin{tabular}{|c|c|c|c|c|}
\hline $\mathrm{n}$ & $\mathrm{k}_{0}$ & $\mathrm{k}_{1}$ & $\mathrm{k}_{2}$ & $\mathrm{~s}\left[\%_{\mathrm{Vol}}\right]$ \\
\hline 1 & -5.76 & 36.84 & 0 & 0.41 \\
\hline $1^{*}$ & -5.89 & 37.27 & 0 & 0.36 \\
\hline 2 & -8.88 & 61.49 & -43.06 & 0.31 \\
\hline $2^{*}$ & -8.45 & 58.80 & -38.14 & 0.25 \\
\hline
\end{tabular}

Tab. 1 shows the calibration parameters $k_{i}$ according to eq. (1) and the average deviation $\mathrm{s}$, which results for each calibration polynomial with grade $n$. The calibration polynomials calculated from low dynamics measurements only, are marked with *, while those calculated from all measurements are not marked. Using a quadratic calibration polynomial and low dynamics measurements only, an average deviation of $0.25 \%$ vol could be obtained over the measuring range between 0 and $10 \%$ vol $\mathrm{CH}_{4}$.

Consequentially, the measurement deviation improves by respecting the conditions and characteristics of measurement dynamics. Thus, using, e.g., a thinner selective membrane with a more rapid response should result in a more appropriate response behavior for the concentration dynamics which was applied.

Taking into account changes of temperature and humidity in the gas flow could lead to further improvements. However, the principle function was successfully demonstrated with an acceptable relative uncertainty of the calibration characteristics. Further steps are validation experiments for different spatial dimensions, considering temperature and water vapor effects as well as the concentration dynamics, and enhancement of the membrane material.

\section{Acknowledgement}

The study was funded by Membranbasierte Gassensoren UG (haftungsbeschränkt) - MeGaSen, Germany.

\section{References}

[1] D. Lazik, S. Ebert, International Journal of Greenhouse Gas Control, 17, 161-169 (2013); doi: 10.1016/j.jggc.2013.04.014

[2] M. Bartholmai, P. Neumann, D. Lazik, Energy Procedia, 37, 4033-4040 (2013); doi: 10.1016/j.egypro.2013.06.303

[3] M. Bartholmai, K.-D. Werner, P. Neumann, S. Ebert, D. Lazik, IEEE Sensors 2014 conference (Proceedings), 942-945 (2014); doi: 10.1109/ICSENS.2014.6985157

[4] P. Neumann, H. Kohlhoff, K.-D. Werner, J. Erdmann, B. Eggeringhaus, M. Kammermeier, M. Schukar, F. Basedau, M. Bartholmai, D. Lazik, S. Ebert, 31st Danubia-Adria Symposium on advances in experimental mechanics (Proceedings), 238-239 (2014);

[5] D. Lazik, S. Ebert, M. Leuthold, J. Hagenau, and H. Geistlinger, Sensors, 9, 756-767 (2009); doi: $10.3390 /$ s90200756 